\title{
Metodología de diagnóstico y evaluación en adquisiciones $y$ procesos técnicos
}

\author{
Cecilia Culebra y Vives* \\ Jaime Pontigo \\ María del Carmen Soto Silis \\ Victor Ocampo Galindo
}

\section{RESUMEN}

Este artículo describe de manera general, el proceso de planeación estratégica. También analiza el diagnóstico efectuado en las áreas de Adquisiciones y de Procesos Técnicos de la Unidad de Información Tecnológica del Instituto de Investigaciones Eléctricas, que se esquematiza en un diagrama causa-efecto, y además presenta la metodología seguida para la ubicación de parámetros estadísticos de evaluación.

Asimismo, alude a resultados parciales de un estudio de tiempos de atención de solicitudes de material bibliográfico, en el que se toma como tiempo de atención, la suma del tiempo de adquisición más el del proceso técnico.

\section{ABSTRACT}

This article describes, in a general way, the process of strategic planning. It also analyzes the diagnosis utilized in the areas of Purchases and Technical processes of the Technological Information Unit of the Institute of Electrical Research which is outlined in a cause and effect diagram. It also presents the methodology followed for the position of statistical parameters of evaluation. In a similar way it alludes to partial results of a study concerning the time used to attend to requests for bibliographical material, in chich the time used to attend to a request is counted as the sum of time used for acquisition as well as for the technical process

\section{INTRODUCCION}

\section{El Contexto Institucional}

a Unidad de Información Tecnológica del Instituto de Investigaciones Eléctricas (IIE) es el ámbito en el que se ha desarrollado este estudio.

El Instituto, creado en 1975, es un organismo público descentralizado, con carácter científico y tecnológico. Su misión es "promover y apoyar la innovación tecnológica en el sector eléctrico, en sus proveedores y usuarios, mediante la investigación aplicada, y los servicios técnicos especializados". ${ }^{1}$
Para llevar a cabo sus funciones el IIE cuenta con más de una veintena de departamentos, organizados en cinco divisiones, $y$ dos Unidades de resultados. Dentro de estas últimas, desde 1992, se cuenta, la Unidad de Información Tecnológica.

La Unidad coordina y centraliza las operaciones de selección, adquisición, organización, almacenamiento, recuperación, transformación, consulta y diseminación de toda aquella información tecnológica que apoye la investigación, el desarrollo y la operación del sector eléctrico del país.
La Unidad, ubicada físicamente en la ciudad de Cuernavaca (Estado de Morelos), es el nodo central de la Red de Información Tecnológica del Sector Eléctrico (RITSE), que interrelaciona a 10 centros del país. Para llevar a cabo sus funciones cuenta con cuatro Coordinaciones de Especialidad: 1) Servicios de Información Tecnológica a la CFE; 2) Servicios de Información Tecnológica al IIE; 3) Servicios Básicos Bibliotecarios; y 4) Asimilación de Nuevas Tecnologías. Adicionalmente, el Gerente de la Unidad tiene apoyo de "Staff" para proporcionar servicios a la industria, para

* Los autores son, respectivamente, coordinadora de Servicios básicos bibliotecarios, gerente de la Unidad de Información Tecnológica, jefa de Proyectos de Procesos Técnicos, e investigador de la Unidad de Información Tecnológica; todos del Instituto de Investigaciones Eléctricas 
gestión administrativa y para procedimientos y automatización.

En los cuatro proyectos de operación que forman la Coordinación de Servicios Básicos, se llevan a cabo actividades de adquisición de documentos y de material monográfico, de adquisición y administración de publicaciones periódicas, y de organización y normalización bibliográficas. ${ }^{2} \mathrm{Su}$ objetivo fundamental es dar servicio y prestar apoyo a las otras áreas de la Unidad, que proporcionan servicio de manera directa a los usuarios.

Dentro de esta Coordinación se ubican los Proyectos de Adquisiciones y Procesos Técnicos que, justamente, son las dos áreas donde se centra la aplicación de la metodología que se describirá a continuación

\section{El Proyecto de Adquisiciones}

En esta área se adquiere y recibe el material bibliográfico, se mantienen relaciones con los proveedores, se factura a los clientes internos y externos y se prepara información dirigida a auditorías contables.

Para llevar a cabo sus funciones, el trabajo se divide en seis actividades: 1) verificación, normalización, ubicación y atención de documentos; 2) verificación, ubicación y precatalogación de acervo monográfico; 3) pagos a proveedores, facturación a clientes y preparación de informes para auditorías contables; 4) colocación y seguimiento de pedidos; 5) recepción y distribución del material; y 6) desincorporación de material bibliográfico (descarte). ${ }^{3}$

\section{El Proyecto de Procesos Técnicos}

En esta área se organiza el material bibliográfico y se crean las condiciones adecuadas de almacenamiento y recuperación de la información, incluyendo aspectos de automatización del proceso y normalización de los datos.

Para llevar a cabo sus funciones, se divide el trabajo en cinco actividades: 1) investigación bibliográfica; 2) análisis bibliográfico; 3) proceso físico; 4) implantación y mantenimiento de archivos; y 5) distribución de material a la Red. ${ }^{4}$

\section{El contexto de Planeación Estratégica}

\section{Definición}

Aunque existen diversas tendencias y definiciones hacia este concepto, nos vamos a referir aquí a la que presentan Giral y González:
"La planeación estratégica en la empresa es una función que intenta maximizar en el largo plazo, los beneficios de los recursos que se dispone mediante la definición clara de misiones, objetivos y metas. La planeación es una función global e integradora, pues para su análisis convergen, prácticamente, todas las áreas de la empresa. Tiene como característica una continuidad que no puede ser acelerada, porque va pasando de la incertidumbre a la decisión, paulatinamente, conforme se va captando información de un entorno que es dinámico y mediante un proceso interactivo en el que se repiten varias veces las mismas actividades, cada vez con mayor precisión" ${ }^{5}$

De acuerdo con este enfoque, los componentes básicos de la planeación estratégica serían: 1) una misión; 2) un medio ambiente; 3) un diagnóstico; 4) una estrategia; 5) una evaluación y 6) un seguimiento.

\section{El Plan Estratégico de la Unidad}

A partir de la misión institucional del IIE, y a raíz de la reunión de planeación estratégica que tuvo lugar en septiembre de 1992, con jefes de proyecto, coordinadores de especialidad y el Gerente de la Unidad, asesorados por el Dr. Enrique Canales, ${ }^{6}$ se estableció un consenso en el que se modificó la misión de la Unidad de Información Tecnológica (UIT), la cual quedó en los siguientes términos:

"Promover y facilitar el uso de la información tecnológica y estratégica, que impulse el desarrollo del sector eléctrico, sus proveedores y sus usuarios, competitivamente". ${ }^{7}$

En un primer acercamiento la problemática general que tendría que afrontarse para llegar a la autosuficiencia económica y a la integración de un plan de mejora continua en la operación de la Unidad, se concluyó que eran cinco los factores fundamentales: 1) el control de calidad; 2) la formación de usuarios; 3 ) la capacitación de recursos humanos; 4) el desarrollo y la adquisición de sistemas y bases de datos; y 5) la adquisición de equipo de cómputo y comunicaciones.

De acuerdo con esta misión y al resto de factores considerados dentro del último Plan Estratégico de la Unidad, se procedió a:

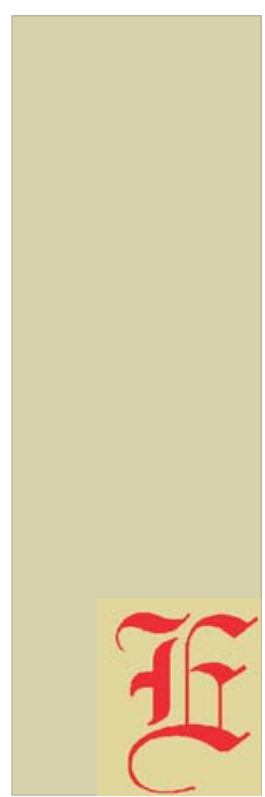

"Promover y facilitar el uso de la información tecnológica y estratégica, que impulse el desarrollo del sector eléctrico, sus proveedores y sus usuarios, competitivamente"

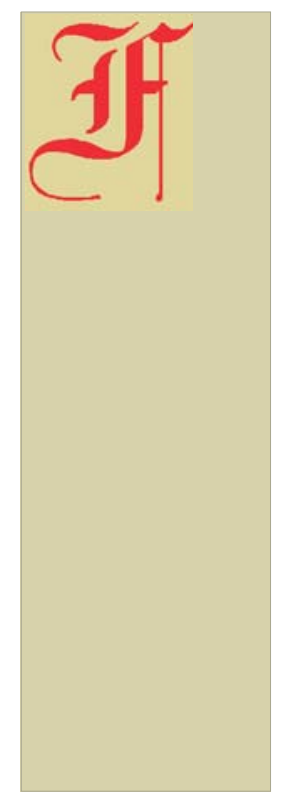


- Elaborar un diagnóstico de la Coordinación de Servicios Básicos, en general.

- Establecer, ubicar y aplicar parámetros de evaluación en los proyectos de Adquisiciones y Procesos Técnicos, en particular.

\section{ELABORACIONDEUNDIAGNOSTICO}

\section{El Diagnóstico: metodología}

Para elaborar el diagnóstico de ambas áreas se utilizaron dos "herramientas" recomendadas para actividades de planeación estratégica y control de calidad:

1) El diagrama sistemático o dendograma (figura 1).

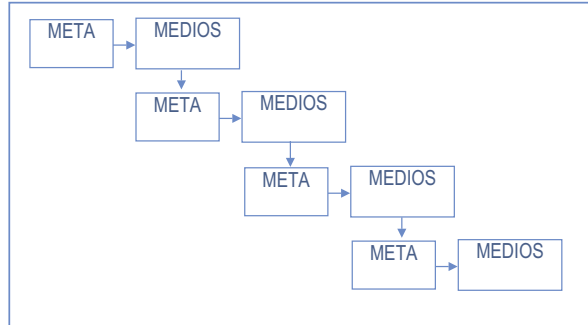

(figura 1)

Bajo este método se establece un objetivo fundamental del área. A partir del mismo, se proponen metas a alcanzar con las cuales se pretende lograr el objetivo fijado. A cada meta, a su vez, se le asignan medios para lograr su consecución. Estos medios, a su vez, se convierten en metas que, consecuentemente, requerirán de otros medios para lograrse. Este ejercicio se concluye cuando se explora hasta la última posibilidad de alcanzar todas las metas. ${ }^{8}$

Es sumamente importante que, para la realización de este ejercicio, participe todo el personal involucrado del área. Además, con la realización de este ejercicio se obtienen algunos beneficios adicionales, tales como:

- Involucrar a los participantes desde el planteamiento del problema, hasta su mismo solución haciendo suyas, de esta manera, las soluciones que surjan en el proceso; los participantes pueden hacer la diferencia de trabajar por una causa, por convicción, en lugar de hacerlo por imposición.

- Hacer claras las delimitaciones de responsabilidad de cada uno de los participantes, en la resolución de los problemas, a la par que se les concede la autoridad y se les presta apoyo para alcanzar dicha resolución (empowerment).

- Clasificar los objetivos y las metas, diferenciándolos de los medios.

- Determinar los recursos humanos y materiales con los que se cuenta para el logro de las metas establecidas.

- Enlistar, jerarquizando, los requerimientos de infraestructura a corto, mediano y largo plazo.
- Servir como base para la planeación anual y la programación mensual y semanal de actividades.

- Integrar el grupo, al identificarse los miembros del mismo, con objetivos y metas comunes.

- Servir como herramienta de comprensión del área, a los investigadores de nuevo ingreso.

- Convertirse en una herramienta de evaluación del desempeño de los participantes, cuyo proceso resulta transparente al evaluador y al evaluado.

Para particularizar, acerca de los factores que estaban afectando la eficiencia interna se trabajó con la segunda herramienta mencionada: el diagrama de causa-efecto.

\section{El Diagrama de Causa-Efecto}

Conocido también como el "diagrama de espina de pescado", o de "Ishikawa", esta es una segunda herramienta que se convierte en un auxiliar valioso para "pensar y resumir" situaciones de diagnóstico. ${ }^{9}$

Básicamente se forma a través de colocar las causas básicas, ya sea de una problemática o de la resolución de las mismas, en las "espinas" principales del diagrama, y a su vez, en cada una de ellas, las subpartes, que la complementen hasta agotar todas las posibilidades. En la figura 2 se muestra el esquema conceptual del uso de esta herramienta.

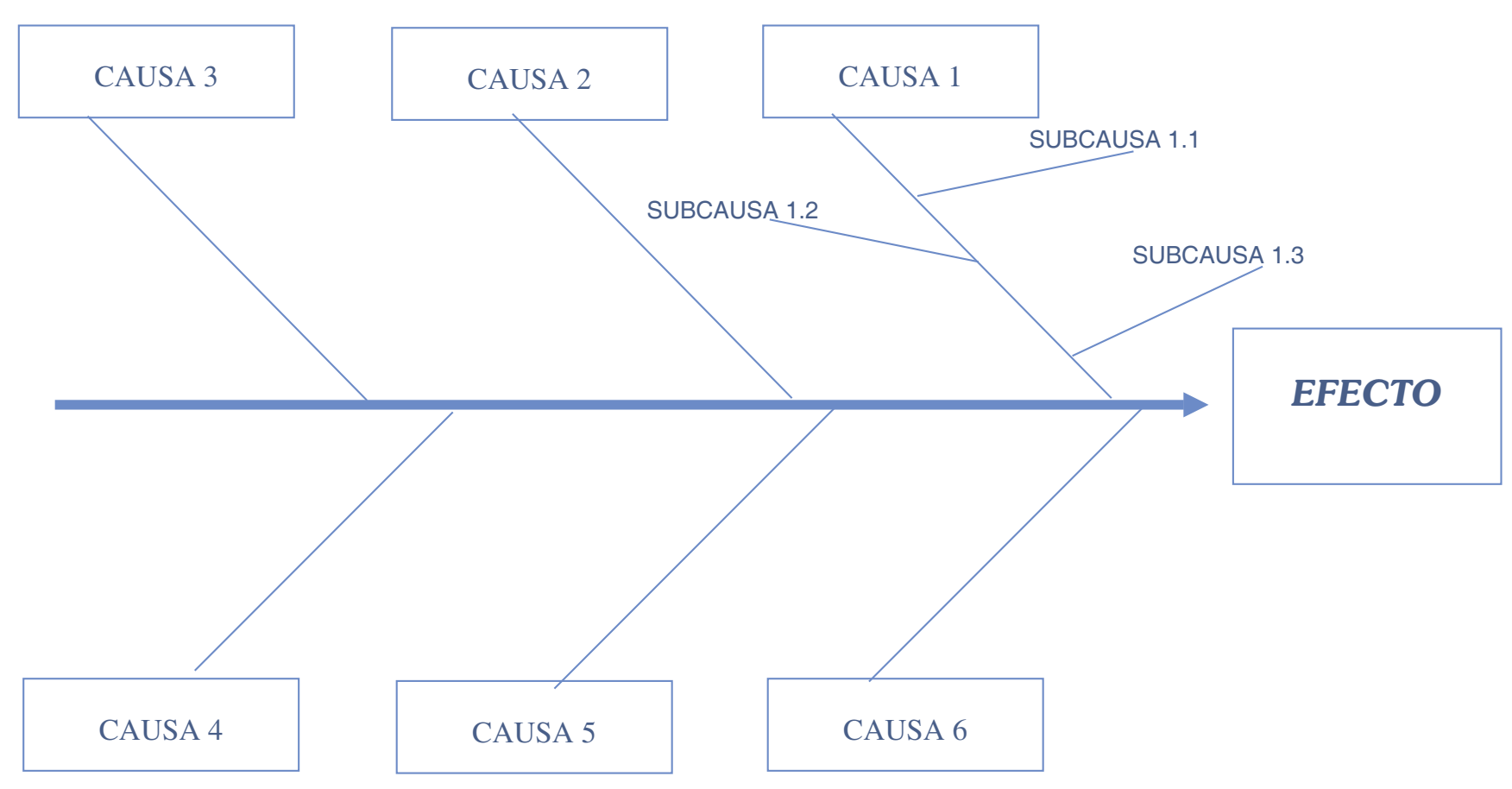

(Figura 2) 
A raíz del uso, de estas dos herramientas, se llegó a establecer, con claridad, un diagnóstico de ambas áreas:

\section{El Diagnóstico Adquisiciones}

En la figura 3 se muestra el diagrama utilizado para hacer el diagnóstico en esta área.

\section{El desarrollo de herramientas de tra- bajo más poderosas}

Particularmente, aquéllas manejadas a través de la computadora, paquetes compatibles para el manejo de información bibliográfica que a la par permitieran un fácil acceso a la información (MICRO-CDS-ISIS); el uso del correo electrónico para interactuar con algunos de los proveedores (ARTTEL-British Library); la implantación de un sistema integral de adquisiciones que incluyera los aspectos de control contable y de información a los clientes; etc.

\section{La optimización de los patrones de búsqueda}

En este rubro se contemplan la separación de la adquisición de monografías y documentos con el objeto de no mezclar tiempos de tramitación cortos, con los largos. La caracterización de las estrategias exitosas de búsqueda y el vaciado de esta información para ser utilizada en un sistema experto, $y$ por último, el procesamiento previo a la compra, de todas las obras cuya adquisición se tramita.

\section{La evaluación de proveedores}

Esto se plantea con el objeto de delimitar una cartera básica de proveedores cuyos servicios mejor se adapten a nuestros propósitos, con base en criterios tales como el tipo de material que proveen, los precios que ofrecen, el tiempo de respuesta, inicial y atención final, el manejo de cuentas (formas de cobranza), los medios de comunicación a través de los cuales se accede a ellos (fax, telex, correo, correo electrónico), y los medios de difusión de sus existencias.

Esta información, una vez recabada, sería la parte medular del sistema experto ya mencionado. ${ }^{10}$

Al contar con patrones de búsqueda optimizados y una cartera de proveedores evaluados, se puede caminar hacia: "hacerlo bien a la primera" como acostumbran decir los japoneses.

La evaluación de herramientas comerciales de verificación y normalización bibliográficas

Este factor incluye la evaluación de obras, cualquiera que sea su formato, catálogos comerciales, impresos, microformatos, discos compactos, etc., con el propósito de mantener una colección núcleo actualizada que permita estar siempre al tanto de lo más reciente.

\section{La implantación de un sistema de ase- guramiento de calidad}

En especial se contempla el identificar sistemáticamente los requerimientos del cliente, enmarcar el sistema dentro del plan estratégico de la Unidad, concientizar y capacitar al personal, ubicar parámetros estadísticos de control de proceso, y diseñar el sistema mismo de aseguramiento de calidad.

Involucrar el área de adquisiciones en actividades, no sólo de la mera obtención de li-

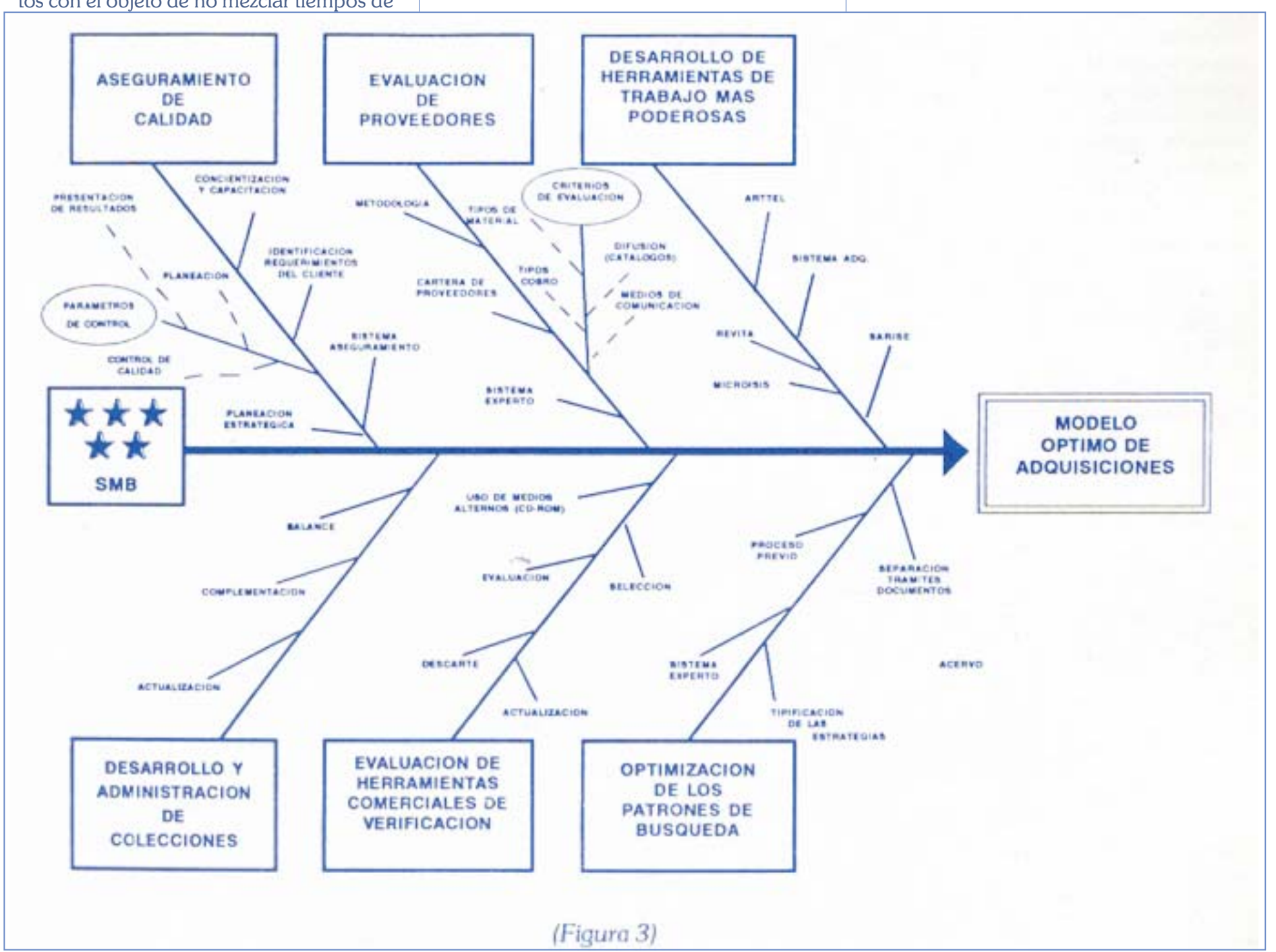




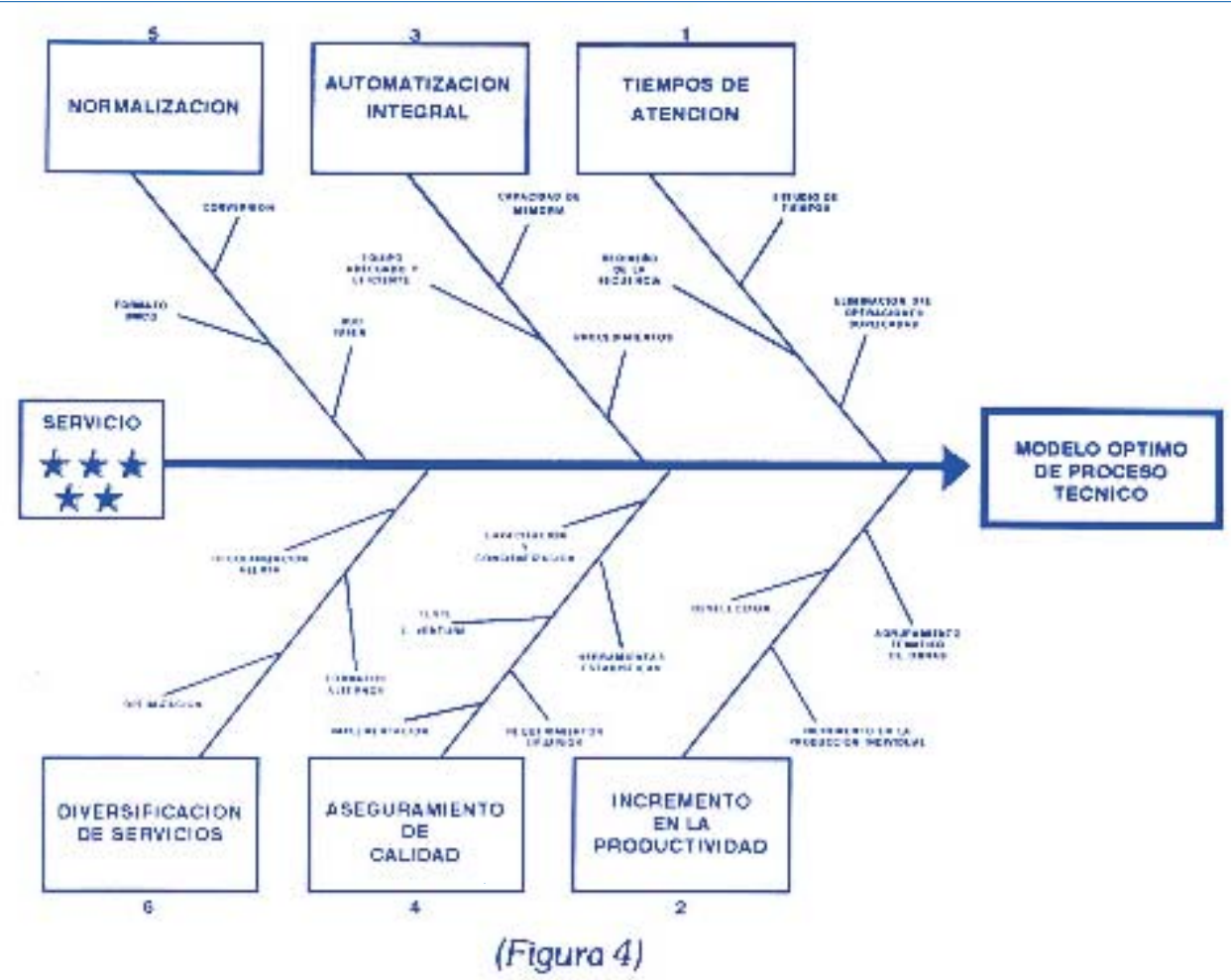

bros y documentos, sino de desarrollo y administración de colecciones.

Implantar un servicio especial al que se denominaría "SERVICIO EXPRESS", en el que independientemente del costo del proceso de adquisición que se genere, se garantice al cliente un tiempo extraordinariamente corto de atención.

\section{El Diagnóstico: Procesos Técnicos}

En la figura 4 se muestra el diagrama utilizado para analizar la problemática en esta área.

\section{Automatización integral}

En este punto se remarcan aspectos de automatización del proceso en su conjunto con el de Adquisiciones, con la intención de digitalizar una sola vez los registros bibliográficos, para irlos migrando en los diferentes archivos e irles agregando "eventos": trámites de adquisición, proceso y finalmente, difusión y préstamo. Aquí se encuentra implícito también el proceso terminal: proceso físico, implementación de catálogos y distribución a la Red.

\section{Asimilación de nuevas tecnologías}

Desde el punto de vista de apoyo a la operación, implicando en ello la calidad y el uso óptimo de recursos.

\section{Normalización de registros bibliográ- ficos}

Se contempla aquí tanto el establecimiento, adopción y validación de normas de operación (políticas), como su aplicación en:

a) compatibilización de archivos;

b) reconversión y mantenimiento de catálogos.

c) implantación de medios para la diseminación del conocimiento de los acervos.

\section{Eficientización interna}

Incluye el análisis de operaciones y de su secuencia a lo largo del proceso; la optimización en el uso de los recursos: la documentación de los cambios, en los procedimientos; y la preparación de la infraestructura necesaria para la implementación de los servicios actuales y potenciales. Una parte muy importante de este factor lo constituye la implantación de un sistema de aseguramiento de calidad, que funcionaría como prueba piloto para el resto de proyectos de la Coordinación.

\section{Diversificación de Servicios}

Abarca la validación de la oferta de los servicios actuales, el diseño de nuevos servicios y la diseminación de ambos, a la Red.

En general, como conclusión de este proceso de diagnóstico, tanto como para el resto de la coordinación, se establecieron tres normas básicas de calidad: tiempo, costo y confabilidad de la información. ${ }^{11}$

En lo que se refiere a la norma de TIEMPO, se procedió a hacer un estudio enfocado a medir tiempos de atención. Dicho estudio se describe con detalle a continuación, tanto en su metodología como en los resultados que se obtuvieron durante la primera etapa.

\section{EL ESTUDIO DE TIEMPOS DE ATENCION}

\section{Antecedentes}

La idea de este estudio, así como su metodología, se ha ido perfeccionando a partir de los trabajos que se mencionan a continuación:

1) "Análisis estadístico de pedidos de material bibliográfico". Trabajo del M.C. Victor Ocampo en el Curso de diseño de Experimentos (IIE, 1987). ${ }^{12}$

2)"El control estadístico como herramienta para el aseguramiento de calidad en la industria de servicios: un caso dentro del IIE". Tesis de Mauricio Gutiérrez. ${ }^{13}$

3) "Parámetros de evaluación de un área de adquisiciones dentro del contexto de planeación estratégica". Ponencia de Cecilia Culebra y Ma. del Carmen Soto, presentada ante el Seminario Anual de la ABIESI, en 1992 (IPN). ${ }^{14}$ 


\section{Premisas}

Las premisas de las que se partieron para la elaboración de este estudio se relacionan directamente, entre otras cosas, con las actividades de verificación e investigación bibliográfica. Hasta agosto de 1990, prevalecían para ambas, las siguientes condiciones:

1.La función de Verificación bibliográfica, ubicada en el Proyecto de Adquisiciones, desarrollaba operaciones relacionadas con la normalización, la verificación y la ubicación de proveedores de TODAS las solicitudes de material bibliográfico (SMB'S), independientemente de que se trataran de trámites de adquisición de documentos o de acervo patrimonial monográfico. Esta función sólo se ejercía, en su fase inicial; el seguimiento y la atención final las llevaba a cabo el Jefe del Proyecto.

2.La función de Investigación Biblio-gráfica, ubicada en el Proyecto de Procesos Técnicos, desarrollaba operaciones relativas a precatalogación y determinación del tipo de proceso que recibiría cada material (duplicado, precatalogación, catalogación especial, catalogación original). Esta función se ejercía sobre todos los materiales del acervo patrimonial después de haberse recibido y registrado, independientemente de su origen y de que, en la mayoría de los casos, ya se hubiera invertido tiempo para su normalización bibliográfica, en Adquisiciones.

A partir de septiembre de 1991, y a raíz del mencionado diagnóstico, se efectuaron los siguientes cambios:

La función de verificación se subdividió en dos:

- Verificación, normalización bibliográfica, ubicación del proveedor, seguimiento y atención terminal de documentos de usuario. Esta función la efectúa un profesional del área de Adquisiciones.

- Verificación, normalización bibliográfica, precatalogación y ubicación del proveedor del acervo bibliográfico patrimonial. Esta función la efectúa un profesional del área de Procesos Técnicos.

La función de investigación bibliográfica, dentro del Proyecto de Procesos Técnicos, se modificó de la siguiente forma:

- Se redujo a la investigación de duplicados en la Red, reportes técnicos, cargos de ejemplares y donativos no solicitados.
- Se amplió a la intercalación del catálogo público del Centro de Información en Palmira, a la actualización de algunos archivos automatizados y a la verificación, previa a su ingreso a las colecciones o a su compra, de donativos y catálogos de editores.

Se implementó el uso de herramientas más poderosas para la verificación bibliográfica, como lo son las bases de datos en disco óptico, y la implementación de rutinas que, tácitamente, conllevan un grado más avanzado de mecanización, como lo es el downloading.

A partir de estas condiciones se elaboró un proyecto de investigación, que formalizara el estudio de tiempos de atención. ${ }^{15}$

De acuerdo con la situación descrita, se determinaron tres premisas definiendo, en cada caso, cuáles habían sido los componentes o factores de preocupación que las habían originado:

\section{Primera}

Sobre el nivel y el número del personal involucrado:

"Con el trabajo de un solo profesional de tiempo completo, y los tiempos parciales de otro profesional y un técnico, se puede llevar a cabo esta operación, en forma correcta, y mejorar además el tiempo de atención"

\section{Componentes}

- Duplicación involuntaria de pedidos

- Duplicidad de operaciones

- Diferencia en los niveles de preparación del personal involucrado

- Confiabilidad de la información

- Tiempos de atención

\section{Segunda}

Sobre la alteración de los procedimientos:

"Se puede invertir el orden de las operaciones y alterar la adscripción de la función, para obtener mejores resultados."

\section{Componentes:}

- Secuencia de las operaciones

- Flexibilidad administrativa respecto de la adscripción de la función

- Mezcla de tiempos de atención

\section{Tercera}

Sobre el uso de nuevas tecnologías:

"Se puede acelerar la atención al usuario y lograr que la información sea más confiable utilizando, en forma adecuada, la tecnología de información"

\section{Componentes:}

- Impacto de la aplicación de nuevas tecnologías

- Adecuación del proceso manual

- Tiempos de atención

\section{Objetivos}

El estudio tiene los siguientes objetivos:

1.Comparar el impacto que se ha tenido sobre los servicios, a raíz de los cambios mencionados, con especial referencia a los tiempos de atención.

2.Detectar, segmentándolo, áreas de mejora en el proceso.

3.Comprobar o no, las premisas planteadas.

\section{Cobertura}

La información que sustenta este estudio, incluye:

1.Solicitudes de material bibliográfico, de usuarios del IIE en Palmira y de enriquecimiento de la colección, generadas en:

- Un primer período que cubre de septiembre de 1989 a agosto de 1990, y

- Un segundo período que cubre de septiembre de 1990 a agosto de 1991 .

2. Solicitudes relativas a material bibliográfico que recibió proceso técnico (monografías, memorias de conferencias, obras de consulta).

3.Solicitudes de las que, reuniendo las dos características anteriores, se localizaron sus hojas de catalogación.

Se excluyeron del estudio materiales producto de adquisiciones especiales, como lo son las membresías y las órdenes continuas, puesto que no se generaron a partir de solicitudes individuales del material, sino por planes generales de compra.

De un total de 1277 solicitudes que reunían las dos primeras características, se localizaron 553 (o sea el $43 \%$ del total de la muestra del tercer inciso). De estas 553, 259 pertenecen al primer período y el resto al segundo. 
Esta muestra se definió a través de la caracterización ya descrita y no al azar.

Las variables que se consideraron para cada uno de estos 553 registros son:

- La fecha en la que el usuario solicita el material.

- La fecha en que se recibe la solicitud de material bibliográfico, en el área de verificación, en Adquisiciones.

- El número de División a que está asignado al usuario que solicita el material.

- El tipo de material.

- El nombre del proveedor.

- La fecha en la que se finca el pedido ante el proveedor.

- La fecha en la que se recibe el material del exterior, en Adquisiciones.

- El número de registro (de adquisiciones o de propiedad).

- El tipo de proceso que recibió el material.

- La fecha de remisión o de envío del material.

\section{METODODLOGIA Y HERRAMIENTAS}

La información se obtuvo en forma directa de dos fuentes:

- Las solicitudes de material bibliográfico atendidas en los períodos señalados.

- Las hojas de catalogación de las obras recibidas coincidentes con las solicitudes, durante el mismo período.

Posteriormente a la compilación física de estas fuentes, se sentaron las condiciones apropiadas para llevar a cabo un análisis estadístico. Para llevar a cabo un análisis completo de esta naturaleza fue necesario integrar un archivo de datos que contuviera la información necesaria para poder hacer diferentes combinaciones de los factores, que se consideraron, podrían afectar el tiempo promedio de atención del material bibliográfico que se solicitó. En este caso, el estudio sólo incluye libros, memorias de conferencias y obras de consulta que son los tres tipos de materiales que reciben proceso técnico.

Los datos de interés para poder llevar a cabo este estudio se encontraban en dos bases de datos distintas, por lo cual fue necesario extraerlos de las mismas auxiliándose del manejador de bases de datos DATATRIEVE TDMS, que funciona en el ambiente VAX/VMS. Estas bases de datos son el "módulo de pedidos" del área de Adquisiciones, que contiene toda la información relacionada con los pedidos de material bibliográfico, datos como: fecha de recepción de la Solicitud del Material Bibliográfico (SMB), fecha de envío del pedido, fecha de recepción del material, proveedor, tipo de material, etc. y la base de datos del área de Procesos Técnicos llamada "remism". Esta última contiene datos relacionados con la catalogación y clasificación del material bibliográfico, datos como: signatura topográfica, tipo de material, número de adquisición, tipo de catalogación (original o duplicada), etc. A su vez, el "módulo de pedidos" contiene dos archivos de datos: el "apedido.dat" y el "apedido1.dat".

Del primero se extrajeron los campos:

SMB (Solicitud de Material Bibliográfico)

FECH-BIBLIO (Fecha de recepción de la SMB en el CIT)

FECH-VERIF (Fecha de verificación de la $\mathrm{SMB})$

FECH-ORDEN (Fecha de ordenado del pedido)

FECH-ATENDI (Fecha de recepción del material

TM (Tipo de material)

STATUS (Status de atendido o cancelado)

DIVISION (Número de la división solicitante)

CLAVE (Clave del proveedor)

Y del segundo, los campos:

REG (No. de adquisición)

SMB (Clave de la SMB, a fin de poder combinar estos campos con los del archivo "apedido.dat")

De la base de datos "remism", del área de Procesos Técnicos se extrajeron los campos:

REG (No. de adquisición: Este dato se obtiene nuevamente a fin de poder enlazar esta información con los datos del área de Adquisiciones)

TC (Tipo de catalogación)

FECHA (Fecha de remisión)
Una vez obtenido el archivo de datos con los campos antes mencionados, se hizo un análisis del mismo por períodos. Se analizó de septiembre de 1989 a agosto de 1990 al que se denominó primer período y de septiembre de 1990 a agosto de 1991 al que se denominó segundo período.

Este análisis de los dos períodos se hizo auxiliándose del paquete estadístico BMDP que funciona en el ambiente VAX/VMS. Se corrió el programa BMDP2D a partir del cual se obtuvo una descripción detallada de los datos, así como una tabla de distribución de frecuencias y un histograma. Se obtuvieron parámetros generales como: media, desviación, estándar, mediana, moda, rango, etc.

Basándose en esta información, se llegó a los resultados que a continuación se mencionan:

\section{RESULTADOS Y CONCLUSIONES}

Siempre teniendo a la vista las tres normas básicas de calidad: TIEMPO, COSTO Y CONFIABILIDAD, el primer acercamiento que se intentó hacer al problema, fue tratar de calcular cuántos días como mínimo, como máximo y en promedio, tarda en ser atendida una solicitud, desde que la origina el usuario hasta que se entrega al Centro de Información receptor.

Con esta perspectiva se obtuvo que el promedio de atención es de 123 días en Adquisiciones para el primer período, y de 109 para el segundo. Sin embargo, para el día 123 , estaban resueltos el $61 \%$ de los casos en el primer período; y en el segundo, para el día 109, sólo el 49\% de ellos.

En el caso de Procesos Técnicos, se observó una situación similar; en tanto que en el primer período se procesó el material en un promedio de 45 días, en el segundo, esto mismo se logró en 25 días. Esto excluye los casos urgentes que se procesan en 24 horas o menos. Sin embargo, para el día 45 estaba procesado el $72 \%$ del material, en el primer período; y en el segundo, para el día 25 sólo el $61 \%$ del mismo.

Al analizar estos datos, se concluyó que por sí mismos no constituyen una herramienta adecuada de control de calidad, puesto que resulta importante asociarles el dato de cuál es el porcentaje de solicitudes atendidas, en lapsos determinados de tiempo.

Para los propósitos de este estudio el tiempo total de atención está constituido por el total de los lapsos comprendidos entre que 
el usuario origina la solicitud, y que se le entrega el material clasificado (figura 5)

Los objetivos del estudio, en su mayoría, se vieron cumplidos:

1. En cuanto a la comparación del impacto en el servicio, producto de los cambios instituidos, una de las evidencias numéricas extraídas del estudio fue que el impacto en la mejora se aprecia más en los tres primeros meses, y decrece hacia los dos últimos. En Adquisiciones, por ejemplo, la relación entre el primero y el segundo período es de 1:1.31 en el primer mes, 1:1.30 en el segundo; baja los dos siguientes (1:1.08 y 1:1.00) y vuelve a repuntar en el quinto mes con una relación de 1:1.20 (figuras 6 y 9).

En el caso de Procesos Técnicos, sucede algo muy similar, a excepción que, la relación va siempre en declive del primer mes, (1:1.23) hasta el último (1:1.03) (figuras 7 y 9$)$.

Tomando el tiempo total del proceso, la relación del primero al segundo período es mucho más significativa, puesto que en el primer mes es de 1:5.59, en el segundo de 1:1.87, en el tercero de 1:1.68, en el cuarto se presenta un declive (1:1.14) y en el quinto vuelve a ser ligeramente mayor que en el cuarto (1:1.23) (figuras 8 y 9)

\section{MODELO DE EVALUACION EN ADOUISICIONES}

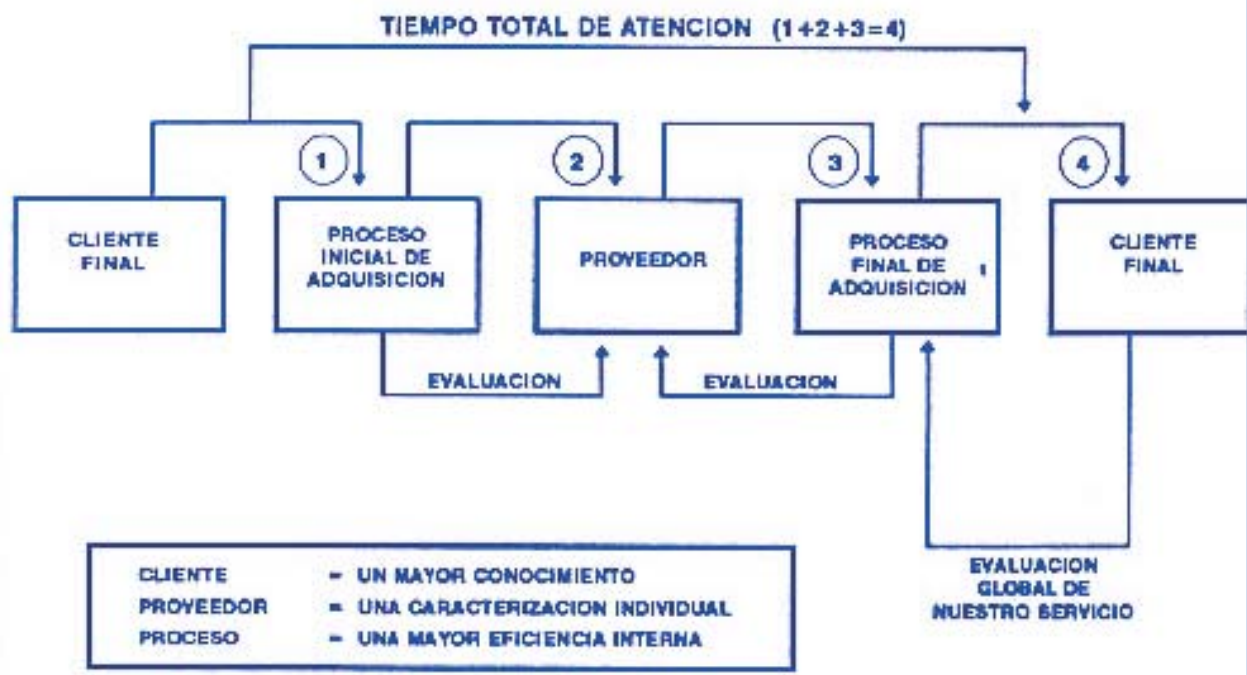

INCLUIDO, EN BU CASO, EL PAOCEEO TECNICO

(Figura 5)

\section{TIEMPOS DE ATENCION DE PEDIDOS \\ DE MATERIAL BIBLIOGRAFICO EN ADOUISICIONES}

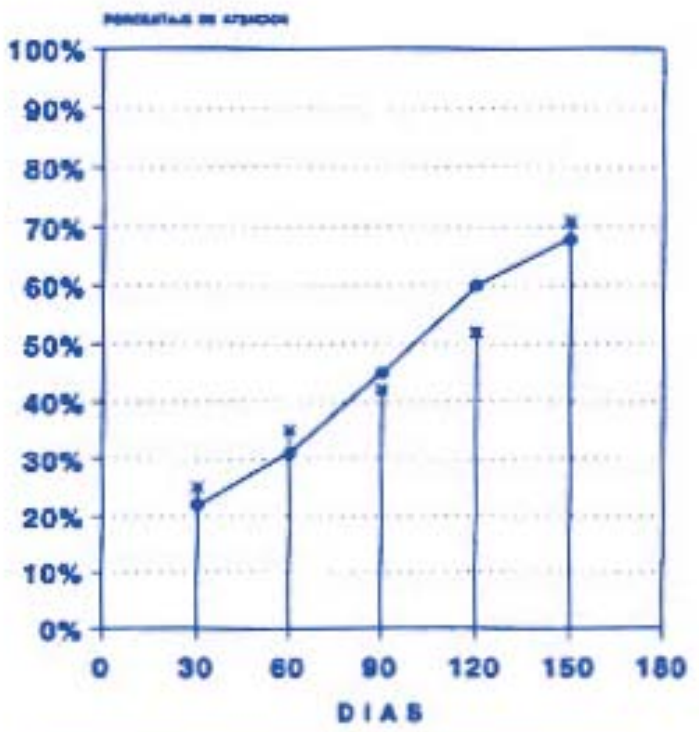

- SEP '
2. En cuanto a la determinación de áreas de mejora en el proceso se detectaron algunos eslabones débiles en la cadena, como son: la primera etapa de verificación bibliográfica, el agrupamiento de los materiales en proceso, en la captura de datos para remisiones y emisión de tarjetas de préstamo y etiquetas, y en el proceso físico.

Por otra parte, algunos otros, se superaron ya, a causa de estos cambios; este es el caso específico de la reducción de adquisiciones duplicadas por error.

3. En cuanto a las premisas planteadas, se puede decir que numéricamente se probaron, sin que por esto se olvide el resto de los factores y de esfuerzos que conforman la totalidad de los tiempos de atención.

Si bien algunos de estos resultados se les puede calificar como "los sobresalientes del estudio" es importante matizar su interpretación por varias causas. La primera de ellas es que aunque se puede decir que se tiene "dominada" la mayor proporción de las operaciones, es en los componentes del final de la curva donde se revierte un mayor esfuerzo, donde se gastan más recursos que, irónicamente, van a ser canalizados sólo para resolver la minoría de los problemas. 
Todos estos casos más difíciles, los que se tardaron más, los que no llegaron, los que causaron irritación en el usuario y frustración en el prestador del servicio, son a los que hay que tipificar, determinando cuáles son aquellos problemas que retrasan la atención de las solicitudes, entre los cuales se tienen: localización de proveedores; material demasiado específico y de no fácil acceso; material muy antiguo y que ya no se edita; por mencionar algunos, prestar atención y sentar las bases de otro comportamiento, una vez que se haya dominado la técnica de obtener esa "mayoría exitosa". Si regresamos a la figura 7 , una situación ideal de operación sería aquélla que en la gráfica refleje la reducción de la pendiente de esa casi "recta" ascendente.

Dicho sea de otra forma, lo que quisiéramos tener sería:

- Muchos pedidos que tarden poco, y

- Pocos pedidos que tarden mucho.

Las estadísticas no pueden tener un papel estático, puesto que el medio que las genera, no lo es; por esta misma razón que no pueden ser interpretados linealmente por sí mismos, sino matizados y ubicados dentro de ese medio ambiente que las generó. En este caso, además de los factores implícitos en verificación e in-

\section{TIEMPOS DE PROCESO TECNICO \\ DE MATERIAL BIBLIOGRAFICO}

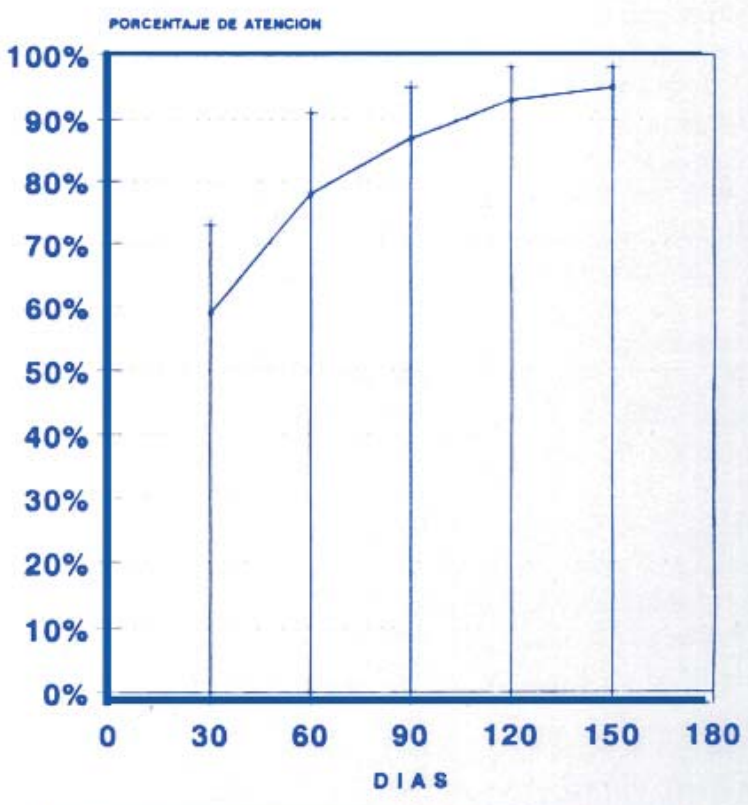

- SEP '89-AGO '90 ${ }^{+}$SEP '90-AGO '91
TIEMPO TOTAL DE ATENCION DE PEDIDOS DE MATERIAL BIBLIOGAAFICO

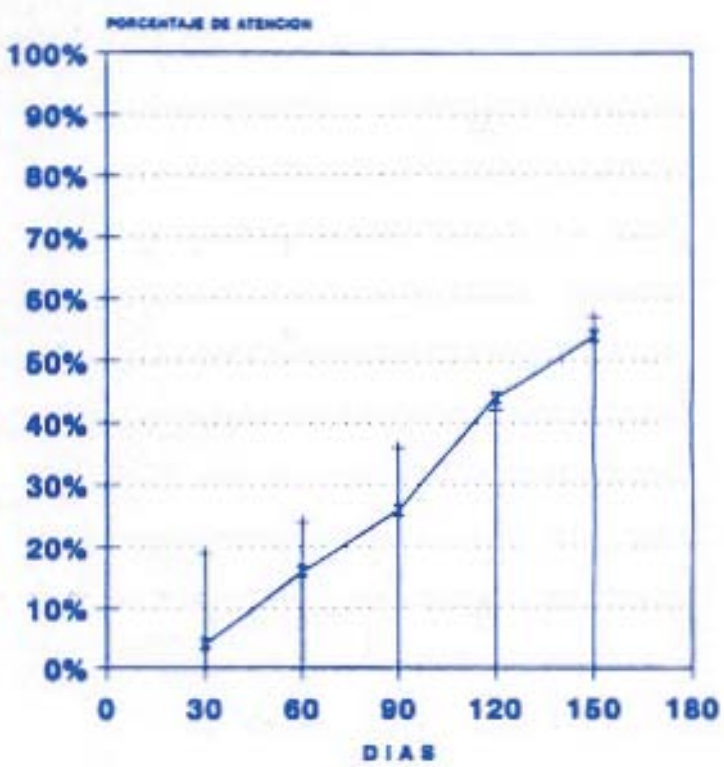

${ }^{2}$ SEP 'BQ-AGO $90^{+}$SEP '90-AGO 91
(Figura 7) vestigación bibliográfica, se han detectado algunos otros factores:

\section{En el Proyecto de Adquisiciones:}

a. En el último año se incrementaron en alrededor de un $85 \%$ las comunicaciones vía fax a nuestros proveedores; este medio ha sustituido paulatinamente al télex y al correo. Además se ha comenzado a tramitar una porción considerable de solicitudes de documentos y otras de menor significación para monografías, por correo electrónico, lo cual agiliza el trámite del material.

b. En este mismo período, se afinaron los procedimientos de reembolso a nuestras cuentas de depósito, regularizándose, por esta razón, el estado de las mismas con un constante "a nuestro favor" en todas ellas. También se automatizó el procedimiento de conciliación de las mismas.

c. A partir de la descentralización administrativa que tuvo lugar en el Departamento, lo que posteriormente desembocó en la formación de la Unidad, se cuenta con la capacidad de pagar con mayor prontitud a nuestros proveedores. Esta situación nos permitió agilizar trámites ante ellos y aumentar su confianza en nosotros, como clientes, de manera notable. 
TIEMPOS DE ATENCION DE PEDIDOS DE MATERIAL BIBLIOGRAFICO

\begin{tabular}{|l|l|l|l|}
\hline \hline \multicolumn{3}{|c|}{ PROCCESO TOTAL } \\
\hline \multirow{3}{*}{ DIAS } & $\begin{array}{l}\text { (PERIODO 1) } \\
\text { SEP.89-AGO.90 } \\
\text { SERIODO 2) }\end{array}$ & $\begin{array}{l}\text { FACTOR } \\
\text { SEP.90-AGO.91 } \\
284\end{array}$ & $\begin{array}{l}\text { DE } \\
\text { RELACION }\end{array}$ \\
\hline 30 & $4 \%$ & $19 \%$ & $1: 5.59$ \\
\hline 60 & $15 \%$ & $24 \%$ & $1: 1.87$ \\
\hline 90 & $25 \%$ & $36 \%$ & $1: 1.68$ \\
\hline 120 & $42 \%$ & $41 \%$ & $1: 1.14$ \\
\hline 150 & $53 \%$ & $56 \%$ & $1: 1.23$ \\
\hline \hline
\end{tabular}

\begin{tabular}{|c|c|c|c|}
\hline \hline \multicolumn{5}{|c|}{ ADQUISICNES } \\
\hline DIAS & 254 & 294 & $\begin{array}{c}\text { FACTOR } \\
\text { DE } \\
\text { RELACION }\end{array}$ \\
\hline 30 & $22 \%$ & $25 \%$ & $1: 1.31$ \\
\hline 60 & $31 \%$ & $35 \%$ & $1: 1.30$ \\
\hline 90 & $45 \%$ & $42 \%$ & $1: 1.08$ \\
\hline 120 & $60 \%$ & $52 \%$ & $1: 1.00$ \\
\hline 150 & $68 \%$ & $71 \%$ & $1: 1.20$ \\
\hline \hline \multicolumn{3}{|c|}{ PROCESOS TECNICOS } \\
\hline \hline DIAS & 254 & 254 & FACTOR \\
\hline 30 & $59 \%$ & $73 \%$ & RELACION \\
\hline 60 & $78 \%$ & $91 \%$ & $1: 1.23$ \\
\hline 90 & $87 \%$ & $95 \%$ & $1: 1.16$ \\
\hline 120 & $93 \%$ & $98 \%$ & $1: 1.05$ \\
\hline 150 & $95 \%$ & $1: 1.03$ \\
\hline
\end{tabular}

Este factor es doblemente importante en las cuentas que no son de depósito y en las que se tiene que pagar antes de recibir el material, lo que nos da la ventaja de control con la oportunidad del material.

d.Se inició el uso de varias bases de datos almacenadas en disco óptico Books in tada por trámites de internación de material, y esto deterioró tanto la relación como los tiempos promedio de atención de este proveedor.

\section{En el área de Procesos Técnicos:}

a. Una de las tres plazas de catalogador, estuvo vacante durante todo el primer periodo del estudio.

b. Se intensificó la tendencia hacia la normalización y la automatización en el área, así como el uso del Bibliofile para aplicaciones de catalogación y emisión de tarjetas.

c. Durante el segundo periodo, la proporción de obras con catalogación en la fuente y con número de LC, fue ligeramente mayor en el segundo período, que en el primero. Es posible que por esta razón el impacto del ahorro de tiempo haya resultado más notorio.

Dentro del proceso de planeación estratégica resulta de suma importancia un diagnóstico previo que permita determinar la realidad actual, para que a partir de ella, se fije la realidad futura que se desee implantar y las estrategias adecuadas para alcanzarla. Para todas estas etapas: diagnóstico, planeación, seguimiento y evaluación, las estadísticas son muy valiosas.

La confiabilidad en la información y la rapidez con la que se presten los servicios, están relacionados en proporción directa con un gran número de factores internos que controlan e intervienen en su conformación. De ahí que resulten valiosos este tipo de monitoreos.

Antes de concluir, queremos hacer patente nuestro reconocimiento al Ing. Alejandro García Alarcón, quien nos asesoró en la formación del archivo de datos.

\section{NOTAS}

1. INSTITUTO DE INVESTIGACIONES ELECTRICAS. Informe anual 1991. Cuernavaca, Mor.: El Instituto, 1992, p. 4

2. "Presentación de la Coordinación de Servicios Básicos Bibliotecarios”. Cuernavaca, Mor.: Unidad de Información Tecnológica, Instituto de Investigaciones Eléctricas, 1992 (Documento de Archivo), p. 5

3. CUlEBRA Y VIVES, Cecilia [y] María del Carmen Soto Silis. "Parámetros de evaluación en un área de adquisiciones dentro del contexto de planeación estratégica". En: Seminario Anual ABIESI, A.C., 1991. p. 5-6 [En prensa] 
4. CULEBRA Y VIVES, Cecilia. "Clasificación del material bibliográfico". En: Primer Seminario Latinoamericano de Información Técnica para el Sector Eléctrico, (Cuernavaca, Mor.: Departamento de Información Técnica, Instituto de Investigaciones Eléctricas, 1987), p. 18-19

5. GIARAL, José [y] Sergio González. Estrategia Tecnológica Integral. México, D.F.: Grupo TecnoPliana, 1988, p. 17

6. CANALES, Enrique. "Planeación Estratégica". Cuernavaca, Mor.: UIT/IIE. Video grabación.

7. UNIDAD DE INFORMACION TECNOLOGICA. "Plan Estratégico de la Unidad, 1992". Cuernavaca, Mor.; UIT/IIE. (Documento de archivo), p.3

8. MIZUNO, Shigero, ed, Management for quality Improvement: The Seven New QC Quality Tools, Cambridge, Ma.: Productivity Press, c1988, p. 143-169.

9. Ibidem, p. 57-59

10. ORTIZ GAMA, Sergio. "Metodología y construcción de reglas de decisión para un sistema experto en la adquisición de material documental". Asesor: Jaime Pontigo (Tesis de Licenciatura de Ingeniería Industrial, Universidad Autónoma del Estado de Morelos, 1988, 192 p.)

11. "Presentación de la Coordinación de Servicios Básicos Bibliotecarios". Cuernavaca, Mor.: Unidad de Información Tecnológica, Instituto de Investigaciones Eléctricas, 1992 [Documento de Archivo], p. 12

12. OCAMPO GALINDO, Víctor. "Análisis estadístico de pedidos de material bibliográfico; trabajo [dentro del] Curso de Diseño de Experimentos". Cuernavaca, Mor.: Departamento de Información Técnica, Instituto de Investigaciones Eléctricas, 1990, [p.v.]

13. Mauricio Gutiérrez Bobadilla. "El control estadístico como herramienta para el aseguramiento de calidad en la industria de servicios: Un caso dentro del IIE". "Aplicación del control estadístico de proceso a un centro de información: El caso del IIE". Asesores: Cecilia Culebra y víctor Ocampo (Tesis de Licenciatura de Ingeniería Industrial, Universidad Autónoma del Estado de Morelos, 1992) [p.v.]

14. CULEBRA, Cecilia [y] María del Carmen Soto, op. cit.

15. CULEBRA y Vives, Cecilia "Estudio de tiempos de atención: Descripción del Proyecto de Investigación". Cuernavaca, Mor.: IIE-DIT, 1991, 5p. [Documento interno] 